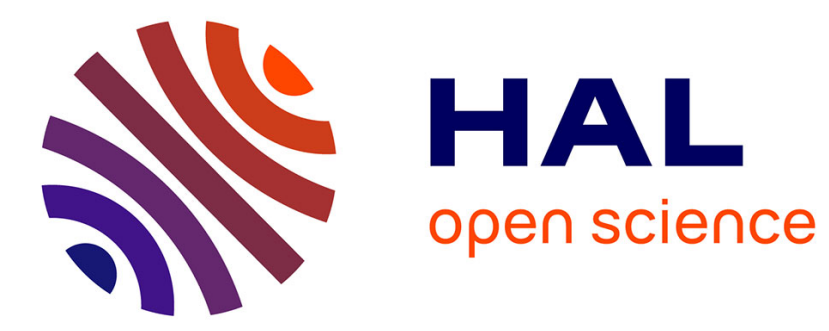

\title{
Micro-scale study of rupture in desiccating granular media
}

\author{
Tomasz Hueckel, Boleslaw Mielniczuk, Moulay Saïd El Youssoufi
}

\section{To cite this version:}

Tomasz Hueckel, Boleslaw Mielniczuk, Moulay Saïd El Youssoufi. Micro-scale study of rupture in desiccating granular media. Geo-Congress 2013, Mar 2013, San Diego, United States. pp.808-817. hal-00835080

\section{HAL Id: hal-00835080 https://hal.science/hal-00835080}

Submitted on 18 Jun 2013

HAL is a multi-disciplinary open access archive for the deposit and dissemination of scientific research documents, whether they are published or not. The documents may come from teaching and research institutions in France or abroad, or from public or private research centers.
L'archive ouverte pluridisciplinaire HAL, est destinée au dépôt et à la diffusion de documents scientifiques de niveau recherche, publiés ou non, émanant des établissements d'enseignement et de recherche français ou étrangers, des laboratoires publics ou privés. 


\title{
Micro-scale study of rupture in desiccating granular media
}

\author{
Tomasz Hueckel $^{1}$, Boleslaw Mielniczuk ${ }^{2,3}$, Moulay Said El Youssoufi ${ }^{2,3}$ \\ ${ }^{1}$ Duke University, Durham, NC 27708, USA; hueckel@ duke.edu \\ ${ }^{2}$ LMGC UMR UM2-CNRS 5508, Université Montpellier 2, 34095 Montpellier Cedex 5, France \\ ${ }^{3}$ MIST Laboratory, IRSN, CNRS UMR 5508, Université Montpellier 2; \\ Boleslaw.Mielniczuk@univ-montp2.fr, Moulay-Said.El-Youssoufi@univ-montp2.fr
}

\begin{abstract}
Capillary bridges between two, three, and multiple fixed glass spheres are examined experimentally during their natural evaporation. The key variables of the process of evolution are measured using a calibrated balance recording and digital image processing with still and high-speed cameras. The calculations of Laplace pressure, as well as suction and surface tension resultant components of the interparticle force are made for two-grain systems. Evolution, properties and failure of evaporating liquid bridge are controlled and induced by decreasing liquid volume. For the two grain configuration, tests show a gradual decrease of suction down to zero and into a positive pressure range before a two step failure occurs, including a formation of a water wire according to a Rayleigh instability pattern followed by a simultaneous rupture at two points of the lowest (negative) total (Gauss) curvature of the bridge surface. For more complex systems, a thin-film pinching instability is shown to result from two-dimensional cavitation of water, leading to a reconfiguration of the water body into separate bridges between individual pairs of grains, which then rupture as described above. Water body instability generated dynamic penetration of air may also provide an imperfection for the granular system potentially leading to cracking.
\end{abstract}

\section{INTRODUCTION}

Evaporation of soil moisture, even during natural convective drying, generates moisture gradients and shrinkage of the material. When shrinkage is restrained, stress (sometimes self-equilibrated) is generated, causing easily cracking when tensile strength is exceeded. Studies of desiccation shrinkage, e.g. by Hu et al. $(2011,2012)$, Kowalski and Mielniczuk (2008), Peron et al. (2009), El Youssoufi et al. (2005), revealed that shrinkage in drying soil develops only when soil is still saturated, while cracks appear shortly after the air entry, i.e. the onset of desaturation, and hence formation and then evolution and rupture of liquid bridges.

The geometry of the liquid/gas interface first enveloping and then withdrawing into the granular body is very complex. Even for an idealized system of monodispersed particles in two-dimensions with a combination of elementary binary, ternary and multiple grain bridges (Urso et al. 1999, Murase et al. 2004) the evolution during evaporation is poorly documented and understood.

In numerous previous studies the capillary forces of liquid bridge between two spherical bodies were measured and estimated numerically at a variety of conditions 
(i.e. Willett et al. 2000, Butt and Kappl 2008, Soulié et al. 2006, Gras et al. 2011). Two grains systems are the most elementary ones, and more articulated multi-grain systems break down eventually to a number of two-grain arrangements. Also three spheres systems were addressed (Urso et al. 1999, Murase et al. 2004, Rynhart et al. 2003). However, most of that work deals with the liquid bridges in static conditions or their behavior during changing of the separation and with a fixed liquid volume, that is without evaporation. Transitioning from a funicular form of the capillary water to a pendular one is also classically recognized, but there are no studies on how such a transition occurs, and what variables possibly control the transitioning.

There are several reasons for which it is essential to determine the conditions for the transition between different configurations of the capillary water, which include the role of the evolving capillary forces in imparting an apparent cohesion to the medium, the instability and dynamic nature of the transition, that may play a role in drying cracking, the advancement of the evaporation process at the moment of bridge rupture, and mass redistribution (if any) following the rupture. In what follows we present selected characteristics of evolution and rupture of binary, ternary, quaternary and other multiple grain planar systems.

\section{EXPERIMENTAL}

Liquid bridges within clusters of two, three and four borosilicate glass spheres (provided by Sandos Fils S.A., with the AFBMA grade 25 and $8 \mathrm{~mm}$ in diameter (3.5 $\mathrm{mm}$ for more than four spheres) were examined. Their centers are fixed in a midplane, while the space between them is filled with distilled water and exposed to evaporation at constant ambient conditions. The changes of the bridge shape and in grain interactions are induced in, and measured by the apparatus shown in Fig. 1.
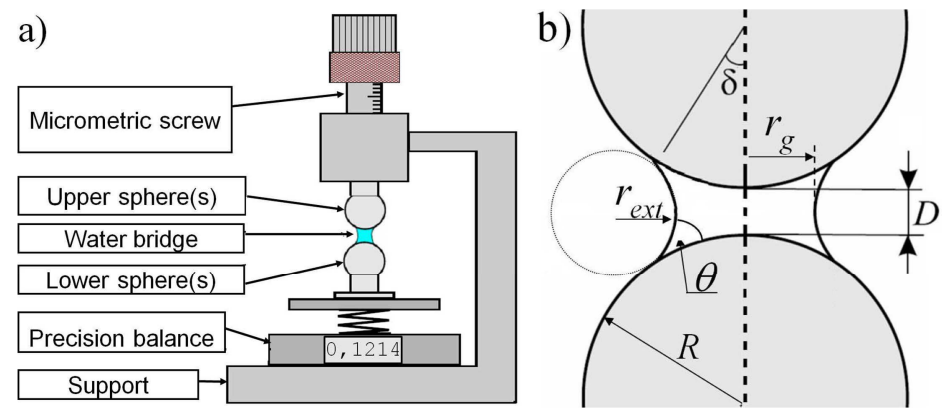

\section{FIG. 1. Apparatus configuration (a) and geometrical parameters of liquid bridge between two spheres (b).}

The bottom sphere(s) is fixed to the substrate and is placed on a high precision balance (with the precision of $10^{-4}$ grams). The top sphere(s) is attached to the micrometer table and suspended from the support without contact with the balance. The vertical separation between the top and the bottom sphere is controlled using a micrometer screw (see Fig. 1) with the accuracy of $0.01 \mathrm{~mm}$. The evolution of capillary during evaporation was investigated at several constant separations, from zero to $0.7 \mathrm{~mm}$ for three and four spheres, and up to $2 \mathrm{~mm}$ for two spheres. After 
setting the requested separation, distilled water is placed into the gap between the spheres with the use of a laboratory micro-syringe, with the accuracy of $0.1 \mu \mathrm{l}$ and the volume of $4 \mu \mathrm{l}$ for 2 spheres, $20 \mu \mathrm{l}$ for 3 spheres and $10 \mu \mathrm{l}$ at 4 spheres configuration, respectively.

Initially, the apparent mass of lower sphere(s) decreases, as a result of buoyancy and the counteraction of the attractive force developing in the liquid bond between the spheres. The attractive force, $F_{C}$, referred to as capillary force is further affected by the evaporation process. It is calculated as $F_{C}=\left(m_{d r y}-m_{\text {exp }}\right) g$, where $m_{d r y}$ is the mass of dry bottom spheres, $m_{\text {exp }}$ is a reading of the balance supporting the bottom spheres and the liquid bridge during drying, and $g$ is the acceleration of gravity. The amount of the evaporating mass of water is measured in a parallel experiment.

A still camera with a macro lens and the resolution of 12 Mpixels is used to image the evolution of the bridge geometry during evaporation. Through image processing it is possible to correlate one-to-one the values of the change in the geometrical properties of the bridge: meniscus radii, contact angle, lateral surface, and the capillary force. To image the bridge at the moment of rupture a high-speed digital camera (Vision Research Phantom v12) was used, with frame rate from 27000 to 42000 frames per second. X-ray microtomography was used to visualize threedimensional shape of liquid bridge between three spheres. The image sequences are processed with Fiji software and with the use of Mathlab@ based program.

\section{RESULTS: BRIDGE SHAPE EVOLUTION}

Variations of bridge volume during evaporation are the main factor affecting the radii of the meniscus surface and the force between the grains. For each examined configuration, different bridge shapes and different evolution can be observed.

Two-sphere bridges. During evaporation the bridge profile evolves with the receding lateral meniscus surface of revolution, following the classical sequence of Plateau (1864). The bridge evolution due to evaporation at $\mathrm{D}=1.3 \mathrm{~mm}$ with the initial liquid volume of $4 \mu \mathrm{l}$ is shown in Fig. 2a. The last instants prior to rupture starting at $2.26 \mu \mathrm{l}$ show a peculiar evolution, in which two strictly conical buttresses develop at the spheres connected by a thin water wire, ab. $70 \mu \mathrm{m}$ in diameter and $170 \mu \mathrm{m}$ in length, which eventually snaps simultaneously after $0.60 \mathrm{~ms}$ (with accuracy up to $1 / 27000$ sec.) at two locations near the wire ends (Fig. 2b). The evolution of the radius of curvature of the gorge at the "equator" plane of the bridge during evaporation is shown for different separations in Fig. 3a. The presented shape of the bridge is to some degree biased by an asymmetry due to the gravity, but it does not seem to alter the main characteristics of the evolution. To quantify the evolution of the water body, we correlate two principal characteristics of the bridges: the radii of their gorge and of the external meridian curvature to the decreasing volume of the bridge liquid.

For larger separations the bridge evolution terminates early with the bridge rupture, with the final gorge radius seemingly independent on separation. For small separations there is a rapid, apparently unstable decrease of the radius near the end of the process. The external radii during evaporation decrease almost as a linear function of the relative volume loss, with slightly higher values for higher 
separations (Fig. 3b). All external radii were measured in the vicinity of the equator, assuming a circular profile locally.

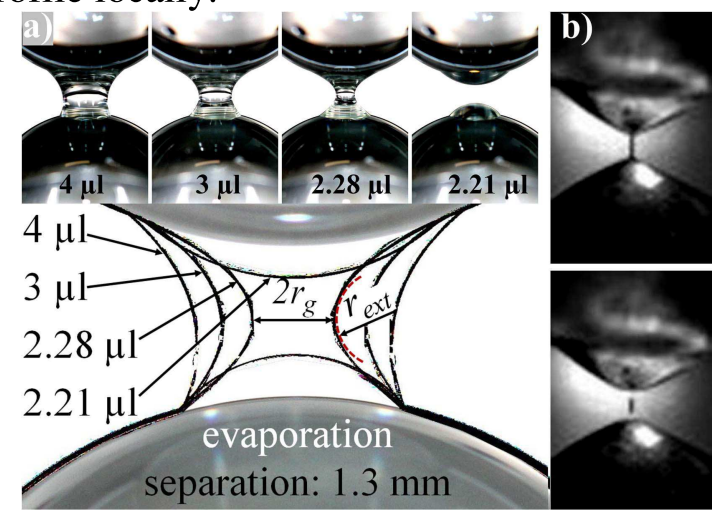

FIG.2. Evolution of evaporating capillary bridge between two spheres at separation of $1.3 \mathrm{~mm}$ with the initial total water volume $4 \mu \mathrm{l}$, at decreasing water volume (in $\mu \mathrm{l})(\mathrm{a})$, detail of the rupture process (fast camera) (b).

a)

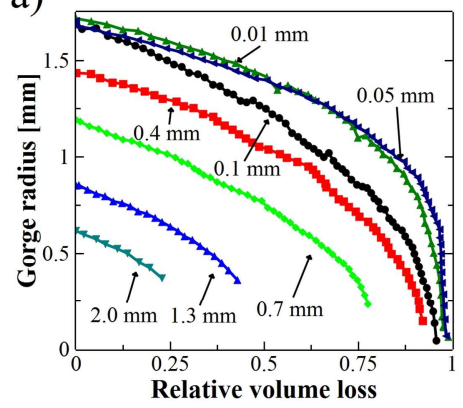

b)

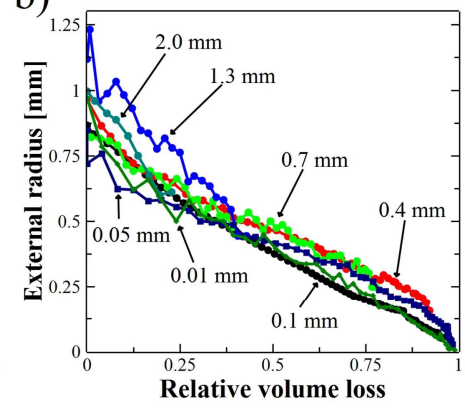

c)

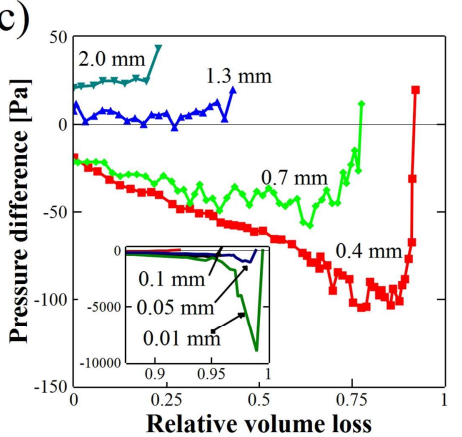

FIG. 3. Evolution of the bridge geometry for two spheres during evaporation with different constant separations: (a) gorge radius; (b) external radius; (c) calculated pressure difference within the liquid bridge.

Notably, prior to rupture the gorge radii become consistently smaller than the external radii. This is of relevance, as it determines the pressure difference that is suction in the bridge water body. The final radii at rupture are measured prior to the onset of the instability of water-wire formation.

\section{Suction and Capillary forces}

During the evaporation tests the total capillary force was measured directly for each separation with the use of the precision balance, (see Mielniczuk et al. 2012a for details). For the two-sphere configuration, it is also possible to estimate capillary forces based on the measured meniscus radii, as a sum of a vertical resultant of the surface tension acting at the perimeter of the neck and the Laplace pressure $\Delta p$ (difference between ambient and internal bridge pressure) as $\Delta p=\gamma\left(r_{g}{ }^{-1}-r_{e x t}{ }^{-1}\right)$ acting at the cross-section of the neck, $F_{C}=2 \pi r_{g} \gamma-\pi r_{g}^{2} \Delta p$ (Willett et al. 2000, Butt and Kappl 2008, Soulié et al. 2006), where $\gamma=0.0496 \mathrm{~N} / \mathrm{m}$ is the experimentally 
determined surface tension of the water actually used. In the calculations the hydrostatic pressure of water acting at the neck level is also considered.

In the paper term suction means a microscopic negative pressure within the body of the individual capillary bridge, calculated as an average of the liquid pressure from the radii of curvature measured at several specific planes of hypothetical symmetry (shown here only for two sphere case). For the bridges between more than two spheres, several averaging procedures are employed. In the latter case, its relation to the macroscopic matric suction is an open issue.

For large separations $(1.3$ and $2 \mathrm{~mm})$ at $4 \mu \mathrm{l}$, the pressure difference is positive (negative suction), and stays that way during the entire process of evaporation until the rupture point, equivalent to $3 \mu \mathrm{l}$ of liquid volume. Positive pressure value occurs in the liquid bridges depending exclusively on the sign and magnitude of both curvatures of the interfaces.

For all considered intermediate and small separations, between $1 \mathrm{~mm}$ and $0.4 \mathrm{~mm}$, the initial liquid bridge is under suction, which gradually increases during evaporation, e.g. to $100 \mathrm{~Pa}$ for $0.4 \mathrm{~mm}$ separation. Then shortly after the suction maximum, at a 1/3rd to 1/4th of the water volume left, there is a sharp decrease of suction to zero with the final pressure reaching positive values up to $20 \mathrm{~Pa}$, before rupture of the bridge takes place. For the smallest separations, i.e. between $0.1-0.01$ $\mathrm{mm}$ (see inset in Fig. 3c) a maximum suction may achieve values as high as $10 \mathrm{kPa}$ at about $10 \%$ of the initial water left, before it sharply drops into positive values of $180 \mathrm{~Pa}$ at $0.1 \mathrm{~mm}$ separation and about zero for $0.01 \mathrm{~mm}$. It is hence concluded that an initial increase of suction is followed by its extensive decline, reaching the zero pressure, and followed by a process of gradual pressurization of the liquid bridge.

In all cases the rupture occurs at a positive pressure. This is quite unexpected, as a vast experimental evidence of an increasing value of suction during macroscopic drying of granular media reaching elevated values at the degree of saturation below 5\% (see e.g. Rahardjo and Fredlund 1995, Peron et al. 2010). It is to be remembered that what constitutes an early stage of loss of water from the capillary bridge, in a granular medium corresponds to a very low saturation degree, related to the entire pore space. However, most of the macroscopic tests are suction controlled, while in our tests suction is a response to evaporation flux.

Nevertheless, adding the suction and surface tension forces up yields the total resultant capillary force, as calculated from the measured radii and hence the contact surface area and perimeter length respectively. The evolution of the calculated total capillary force during evaporation is shown in Fig. 4a and compared to the directly measured force. The total capillary force during evaporation results to be tensile all along. The values of pressure difference of Fig. 3c became magnified by the cross section surface area of the gorge at the beginning of the process, but substantially diminished toward the end when the gorge area becomes very small, when calculating the resultant force. In the terminal phase, as the contact area becomes very small, the trend of the surface tension force prevails over the suction force (for the considered volume of liquid). The comparison with the directly measured force is more than satisfactory, with some exception for 0.1 and $0.01 \mathrm{~mm}$ separation. This is an indirect confirmation that adopted experimental techniques are sufficiently accurate for the present purpose, at least for separations $>0.1 \mathrm{~mm}$. 

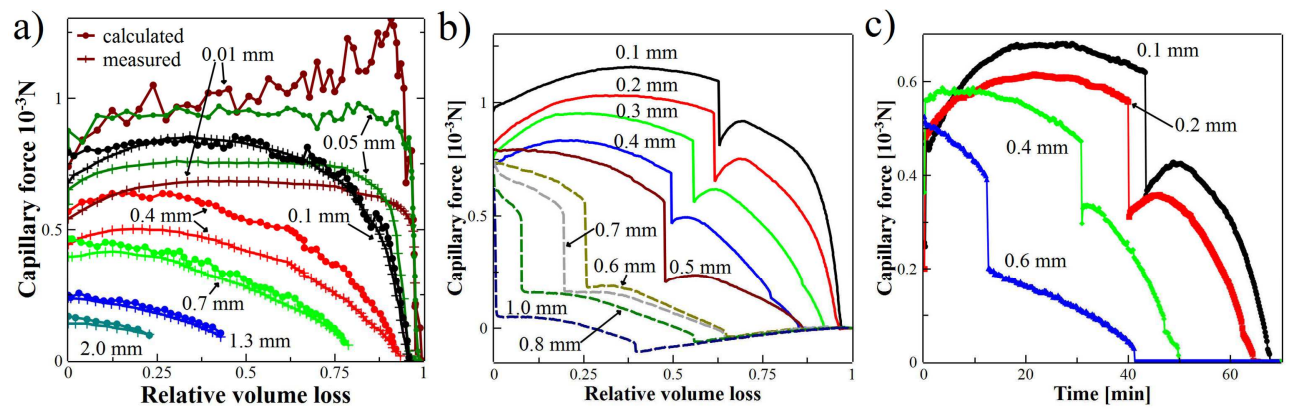

FIG. 4. Evolution of the total resultant capillary force between two spheres during evaporation, as calculated from the radii of curvature and measured directly in the experiment (a); and measured for 3 spheres (b) and 4 spheres (c).

Three sphere bridges. For a three-sphere configuration, the initial 3D, three-branch capillary water body evolves during evaporation in two distinctly different modes of transformation/rupture, referred to as symmetric and non-symmetric mode, depending on the "projected separation" (non-dimensional), as found empirically. Projected separation is conceived as a measure projected on the axis of symmetry (AA in Fig. 5a) of the distance between the position (center, or any other characteristic point) of the top (undeformable) sphere at (symmetric) contact with the other two spheres and that at a separated position, divided by the sphere diameter. In the considered cases of $0.8 \mathrm{~mm}$ and $0.35 \mathrm{~mm}$ diameter spheres, a threshold values were found of about 0.075 and 0.0714 , respectively, below and above which a symmetrical or non-symmetrical evolution and rupture would take place. As a result of the two modes of rupture, either three (for symmetric mode) or two (for nonsymmetric) independent pendular bridges are produced.

The initial phase of the development of the symmetric mode consists in an inward motion of what is seen in the planar projection as a motion of lateral menisci. More difficult, but also more significant to observe, is the evolution and inward motion of the central (symmetric) menisci on both sides, orthogonal to the bridge plane (front and back). This region of the bridge resembles two sheets of the surfaces spanned on the spheres, with a deflating volume between them, as shown in Fig. 5b-d. The shape of cross-section of capillary bridge at separation of $0.7 \mathrm{~mm}$, with the volume of 20 , 15 and $11 \mu \mathrm{l}$, determined with x-ray microtomography, is presented in Fig. 5c. This sequence simulates the evaporation process, as the water volumes were injected between spheres (i.e. they were not achieved through evaporation), but the localized changes in water body thickness can be well seen. It is postulated that as the faces of the water body approach and come eventually together to form a thin film undergoing a pinching instability (Vaynblat et al. 2001), as observed experimentally by Maeda et al. 2003.

The transformation from funicular state to three pendular bridges is observed as a jump on capillary force curve (Fig. 4b) and involves transition through unstable funicular states, see Childs 1969, occurring within 1/300 sec, as shown in Fig. 5e. Possible theoretical configurations of the liquid bridge produced between three spheres were dicussed by Urso et al. (1999). 

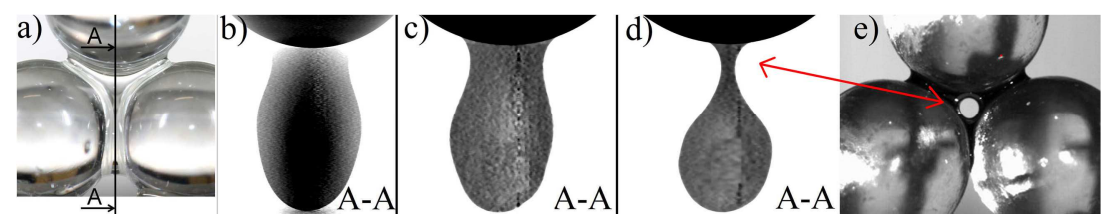

FIG. 5. Cross-section plane of capillary bridge (a); $x$-ray tomography of liquid bridge between three spheres with separation of $0.5 \mathrm{~mm}$ and water volume of 20 $\mu \mathrm{l}(\mathrm{b}), 15 \mu \mathrm{l}$ (c) and $11 \mu \mathrm{l}(\mathrm{d})$; (e) an expanding 2D gas bubble, caused by a local thin film instability

At higher projected separations (over 0.07) the initial symmetric evolution of the two lateral menisci evolves in a abrupt manner into a non-symmetrical pattern, with a significant difference between rates of the left and right meniscii observed, favouring one of them, that proceeds with an unstable rate until its disappearance. At the end, the situation stabilizes and two separate pendular bridges are created. This transformation includes a vigorous transport of water from the moving part to the rest of the bridge. The subsequent evolution into two pendular states is much slower. This mode of rupture is presented in Fig. 6.

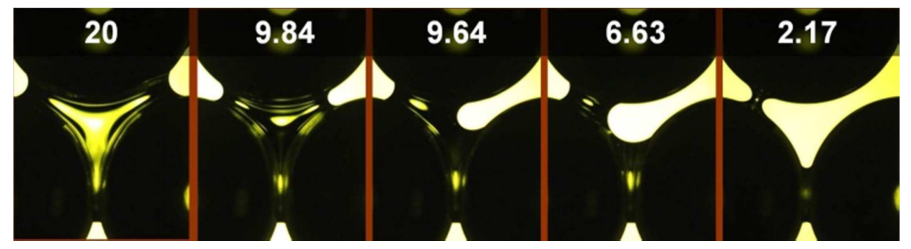

\section{FIG. 6. Evaporation of capillary bridge between 3 spheres, 20 microliters of water, distance of $0.7 \mathrm{~mm}$, lateral water movement.}

Other grain arrangements were also examined, with 4 to 8 spheres on one plane, at different projected separation between spheres (horizontally, the spheres were in contact). The evolution during evaporation process includes appearance of forms of instability presented above, depending on the grain arrangement and their projected separations. At smaller separations capillary bridge the evolution is symmetric, it lasts less than 1/300 s and leads to arising of isolated pendular bridges. Internal gasliquid interfaces in general move faster, than external ones. For the higher separation it is possible also to observe the second described mode of bridge rupture and water transport. Both types of evolution are presented with several examples in Fig. 7.

For the arrangements of three and four spheres the progress of capillary force is presented in Fig. 4b,c. In all cases a substantial capillary force jump of up to $50 \%$ of the initial value is recorded. For the distances smaller than $0.7 \mathrm{~mm}(0.4 \mathrm{~mm}$ for four spheres), increase of force after rupture may be observed, small and short for 0.5 to $0.3 \mathrm{~mm}$, but significant and longer (over 20 minutes) at lower separations.

For the separations of over $0.6 \mathrm{~mm}$ the force decreases monotonically, and capillary bridge very soon reconfigures to funicular state and next to two pendular bridges. Sudden decrease of force by about $0.2 \mathrm{mN}$ is measured. Next the force value 
continues decreasing (slower than previously) and pendular bridges break after an almost linear decrease of force value, as seen as a step on the graph.

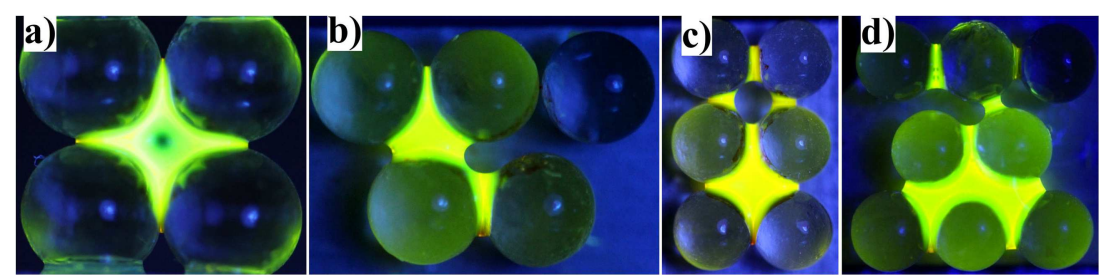

Fig. 7. Two modes of evaporation of water bridge in different spheres arrangement, (a), (c) expanding gas bubble, (b), (d) transport of water mass.

\section{Rupture}

The rupture of the bridges between two spheres occurs after a relatively slow evolution in the first stage of evaporation with the bridge suddenly developing into a very thin water wire; which next ruptures simultaneously near both ends within 0.3$0.9 \mathrm{~ms}$. Then ruptured wire transitions into a small droplet of water, which free-falls in the gravity field (Fig. 8). Eventually the rest of the mass of water is separated into two drops attached to each of the spheres. For separation of $D=1.3 \mathrm{~mm}$ (Fig. 8b) bridge ruptures at the water volume $V=2.28 \mu \mathrm{l}$, with the water wire $90 \mu \mathrm{m}$ in diameter, and $130 \mu \mathrm{m}$ in length. At other separations length of water wire is from 50 to $150 \mu \mathrm{m}$, with the diameter from 70 to $130 \mu \mathrm{m}$.

For four, six and more spheres arranged in one plane, the capillary bridge ruptures in several different ways, following the same patterns as for three spheres, either in symmetric (i.e. Fig. 7a and c) or non-symmetric mode (i.e. Fig. 7b and d).

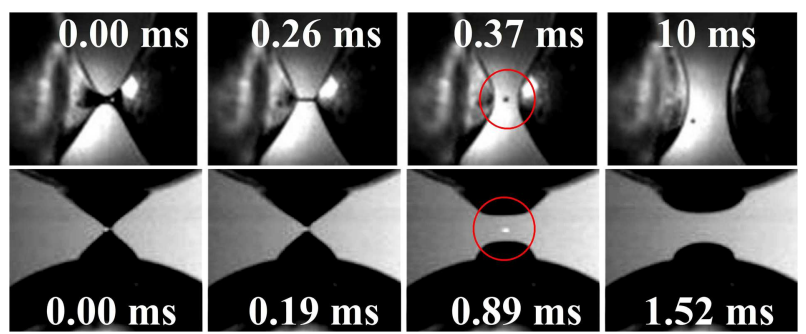

FIG. 8. Photos of cracking capillary bridge in vertical (a) and horizontal (b) configuration (made with high speed camera). Small droplet of water is visible for both configurations.

The formation of the vapor bubble, can be viewed as a liquid cavitation (as described for plants by Cochard 2006). For micro- and nanoscopic separations Maeda et al. (2003) found that density of the matter within the center of the thin film is half way between that of liquid and its vapor. Such an instability process may be also understood in terms of stretching forces, exceeding the strength of the water film (see Vaynblat et al. 2001). 
The second (non-symmetric) mode of rupture is observed at higher separations also for multiple spheres. At such case the movement of the meniscus is similar to the described above three-sphere systems, with a much higher rate, than during the stable phase, but slower than eruption of gas bubble.

Notably, because of the 3-D character of the water body there is much of energy expended to create a large amount of the new surface, and to displace significant volume of the liquid. These issues call for further studies.

\section{CONCLUSIONS}

The general aim of the studies was to identify the mechanisms and critical variables that control the process of the evolution of the water body within capillary bridges during the process of their evaporation. A significant negative pressure (suction) and also a negative total resultant capillary force acting (pulling) the grains are observed at the beginning of the process. The presence of a positive pressure in the terminal phase of evaporation implies that there is a repulsion exerted by water against the grains, but attractive surface tension force is much higher so that the total capillary force remains attractive. Clearly, the positive pressure within capillary bridges prior to their rupture constitute a substantially new development for our understanding for the unsaturated soil mechanics. Rupture of the bridge takes place via pinching of the water column evolving into a water wire, which next breaks simultaneously at two points. This phenomena occur as an instability, developing within 1/3000 second.

For liquid bonds between three and more spheres their behaviour and evolution is dependent on the separation and may be either symmetric or non-symmetric. In the first scenario the gas-liquid interfaces are converging toward the center of the system, to eventually form a suspended thin liquid film. Then the film breaks at a very high rate forming an expanding gas filled hole, until its boundary reaches the walls of the glass spheres. Subsequently, the water body reconfigures and transforms into separate, toroidal bonds, which then slowly shrink, as the two-sphere bridges. The non-symmetric mode involves an accelerated water transport between segments of the bridge as a result of formation of new free surfaces deep within the granular body. Significant jump in capillary forces can be seen in both cases. These two scenarios constitute possible micro-mechanisms of air entry into saturated soils, depending on local grain configuration.

No reasonable numerical criterion for rupture has been established via material properties of water. However, instability of the liquid bridge in the form of pinching or thinning the bridge is a most likely an explanation of the observed and measured quantities in this exercise. Its prediction requires analysis, which takes into account the rate of evaporation of water and possibly disjoining pressure.

\section{ACKNOWLEDGEMENTS}

The authors acknowledge the partial support of US NSF grant \#0700294 of CMMI Division, Geomechanics and Geomaterials Program (T. Hueckel) and support of CNRS, MIST laboratory and the Languedoc-Roussillon region (B. Mielniczuk, M. S. El Youssoufi, T. Hueckel). 


\section{REFERENCES}

Butt, H-J., Kappl, M. (2008). "Normal capillary forces.” Adv. Colloid Interface Sci. 146:48.

Childs, E. C. (1969). An Introduction to the Physical Basis of Soil Water Phenomena. Wiley-Interscience, London.

Cochard, H. (2006). "Cavitation in trees." C. R. Physique 7:1018.

El Youssoufi, M. S., Delenne, J. Y., Radjai, F. (2005). "Self-stresses and crack formation by particle swelling in cohesive granular media." Phys. Rev. E 71: 051307.

Gras, J.-P., Delenne, J.-Y., Soulié, F., El Youssoufi, M.S. (2011). "DEM and experimental analysis of the water retention curve in polydisperse granular media." Powd. Tech. 208:296 (2011).

Hu, L.B., Peron, H., Hueckel, T., Laloui, L. (2012). "Desiccation shrinkage of nonclayey soils: multi-physics mechanisms and a microstructural model." Int. J. Numer. Anal. Meth. Geomech, online.

Hu, L.B., Peron, H., Hueckel, T., Laloui, L. (2012). "Desiccation shrinkage of nonclayey soils: a numerical study." Int. J. Numer. Anal. Meth. Geomech., online.

Kowalski, S.J., Mielniczuk, B. (2008). "Analysis of effectiveness and stress development during convective and microwave drying." Dry. Technol. 26: 64.

Maeda, N., Israelachvili, J. N., Kohonen, M. M. (2003), Proc. National Academy of Sciences of the USA, 100(3):803.

Mielniczuk, B., Hueckel, T., El Youssoufi, M. S. (2012a). "Evolution of capillary bridges between two spheres. Experiments.", submitted.

Mielniczuk, B., Hueckel, T., El Youssoufi, M. S. (2012b). "Evolution of capillary bridges between two spheres. Analysis and Implications", submitted.

Murase, K., Mochida, T., Sugama, H. (2004). "Experimental and numerical studies on liquid bridge formed among three spheres." Gran. Matter 6:111.

Peron, H., Delenne, J. Y., Laloui, L., El Youssoufi, M. S. (2009). "Discrete element modelling of drying shrinkage and cracking of soils." Comput. Geotech. 36: 61.

Plateau, J. (1864), The Ann. Rep. of the Smithsonian Institution, Wash. DC, 338 p.

Rahardjo, H., Fredlund, D. G. (1995). "Experimental verification of the theory of consolidation for unsaturated soils." Can. Geotech. J. 32:749.

Rynhart, P. R., McLachlan, R., Jones, J. R., McKibbin, R. (2003). "Solution of the Young-Laplace equation for three particles." Res. Lett. Inf. Math. Sci. 5:119.

Soulié, F., Cherblanc, F., El Youssoufi, M. S., Saix, C. (2006). "Influence of liquid bridges on the mechanical behaviour of polydisperse granular materials.", Int. J. Numer. Anal. Meth. Geomech. 30:213.

Urso, M. E. D., Lawrence, Ch. J., Adams, M. J. (1999). "Pendular, funicular and capillary bridges." J.Coll. Interf. Sci. 220:42.

Vaynblat, D., Lister, J. R., Witelski, T. P. (2001). "Rupture of thin viscous films by van der Waals forces: Evolution and self-similarity." Phys. Fluids 13( 5):1135.

Willett, Ch.D., Adams, M. J., Johnson, S. A., Seville, J. P. K. (2000). "Capillary bridges between two spherical bodies." Langmuir 16:9396. 